\title{
Strategy of Material Parameters Identification for Non Linear Mechanical Behavior: Sensitivity of FE Computation
}

\author{
S. Bouvier ${ }^{+}$L. Alves ${ }^{*}$ and A.M. Habraken ${ }^{++}$ \\ ${ }^{+}$LPMTM-CNRS, UPR9001, University Paris 13, $99 \mathrm{av} . J-B$. Clément, 93430 Villetaneuse, France \\ "Dept. of Mech. Engineering, University of Minho, Campus de Azurèm, 4800-058 Guimarães, Portugal \\ ${ }^{+}$ArGEnCo Dept., University of Liege, Chemin des Chevreuils 1, 4000 Liege, Belgium
}

\begin{abstract}
The purpose of the present work is to analyze several aspects related to the connection between the constitutive models, their identification and the FEM predictions. Several issues are addressed: the experimental data base that should be used in the identification procedure, the choice of the mechanical tests involved (monotonous and/or non-proportional loading, homogeneous or heterogeneous tests...), the identification strategies (direct or inverse FE optimization, simultaneous or sequential material parameters identification...). Besides its obvious interest, such study aim to find a good balance between the number and the type of relevant involved mechanical tests in material behavior characterization. This is an important issue for industrial applications.
\end{abstract}

Keywords: Non-Linear Behavior, Anisotropic material, Constitutive laws, Sensitivity analysis, Identification.

PACS: Replace this text with PACS numbers; choose from this list:

http://www.aip.org/pacs/index.html

\section{INTRODUCTION}

In the context of numerical accuracy requirement, the hardening laws and yield criteria used to describe the material behavior play a very significant role. In recent years, intensive efforts have been done in order to develop new constitutive models that allow a more accurate description of the mechanical behavior of metal sheets. However, one should emphasize the fact that the question of the suitable way for material parameters identification is not completely solved so far. The purpose of the present work is to analyze several issues related to the connection between the constitutive models, their identification and the FEM predictions. Several practical applications are presented and discussed where we mainly focus on the effect of the identification strategies, on the numerical simulations results. The sensitivity of the FE results to the modeling description was discussed in [Bouvier et al., 2006]. The first problem compares different strategies of yield locus identification and their effect on ears prediction in deep drawing. The second investigated problem analyses two identification approaches: (i) direct identification (i.e. local volume element computation) developed in University Paris 13, (ii) inverse identification using FE

CP907, 10 th ESAFORM Conference on Material Forming, edited by E. Cueto and F. Chinesta (C) 2007 American Institute of Physics 978-0-7354-0414-4/07/\$23.00 
computation, developed in University of Liege. The third problem deals with the sensitivity of FE computation to the type of mechanical data involved in the identification process.

\section{YIELD SURFACE IDENTIFICATION}

The initial and the induced anisotropy in materials are described through the shape, the size and the position of the yield locus. For well-annealed materials, it is rather admitted that their crystallographic textures are responsible for their initial anisotropy. The latter define the shape of the yield surface. Therefore, sequential strategy based on the identification of the yield surface material parameter using only the initial anisotropy (i.e. crystallographic texture, in-plane strain and/or stress anisotropy) seems to be quite acceptable. The hardening material parameters are identified on the stressstrain curves with fixed yield surface parameters. In this context, several approaches can be compared. An example is presented in Figure 1(a) in case of [Hill, 1948] criterion. The labels indicated on the figure mean:

(1) Taylor-Bishop-Hill model (TBH): predicted $\mathrm{r}(\alpha)^{1}$ using TBH model and experimental crystallographic texture.

(2) Experimental data: measured $\mathbf{r}(\alpha)$ using uniaxial tensile test along different orientations with respect to the rolling direction.

(3) [Hill, 1948] using mechanical data: identification of [Hill, 1948] parameters $\{\mathrm{F}, \mathrm{G}, \mathrm{H}$ and $\mathrm{N}\}$ using the measured $\mathrm{r}(\alpha)$.
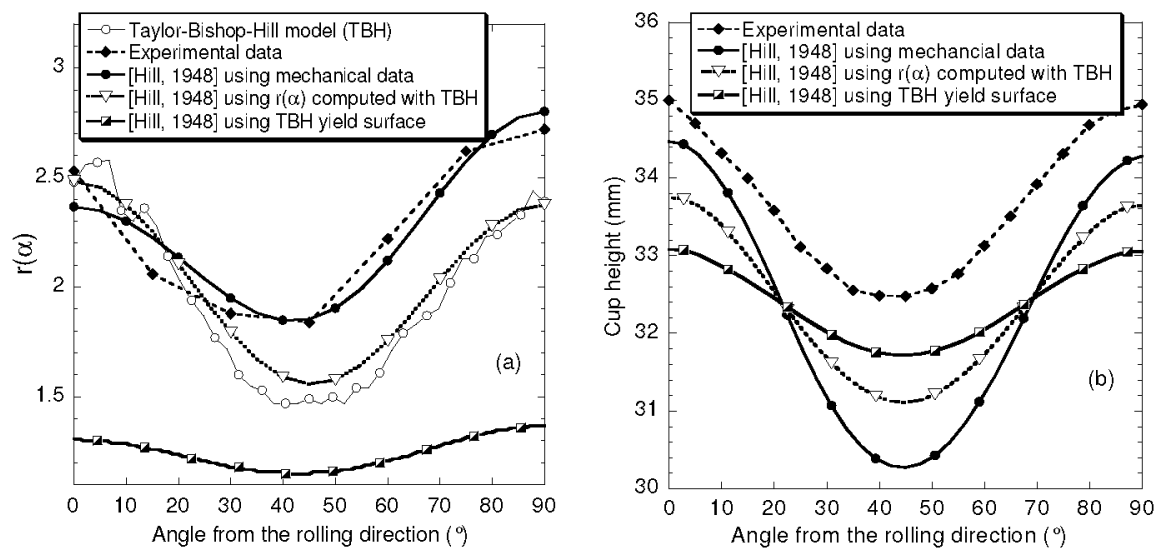

FIGURE 1. (a) Different strategies in the identification of the material parameters for the quadratic [Hill, 1948] yield criterion. (b) Ears prediction using the previous identified material parameters in deep drawing of a cylindrical cup.

[Hill, 1948] using $\mathrm{r}(\alpha)$ computed with TBH: identification of [Hill, 1948] parameters $\{\mathrm{F}, \mathrm{G}, \mathrm{H}, \mathrm{L}, \mathrm{M}, \mathrm{N}\}$ using TBH predicted $\mathrm{r}(\alpha)$.

${ }^{1}$ Hill coefficient of anisotropy. 
[Hill. 1948] using TBH vield surface: identification of [Hill, 1948] parameters $\{\mathrm{F}, \mathrm{G}, \mathrm{H}, \mathrm{L}, \mathrm{M}, \mathrm{N}\}$ using best fit of [Hill, 1948] yield locus to the TBH one.

As general comments: (i) there are some discrepancies in the predicted $r(\alpha)$ using the TBH model and the mechanical data; (ii) the predicted $\mathrm{r}(\alpha)$ using the last strategy (i.e. yield surface fitting) is relatively far from the others. In this situation, the identified material parameters describe the whole anisotropy of the material. On the contrary, the identified material parameters using the mechanical data reduce the material anisotropy to the in-plane one.

The identified parameters are used in the simulation of a deep drawing of a cylindrical cup (Figure 1(b)). The results clearly show a significant sensitivity of the FE predictions to the strategy of identification. An improvement of the predicted solution is obtained when the whole material anisotropy is described.

\section{SEQUENTIAL VS SIMULTANEOUS MATERIAL PARAMETERS IDENTIFICATION}

The material parameters identification of constitutive laws requires the use of mechanical tests suitable for the behavior under investigation. Such mechanical tests provide the global response (e.g. force-displacement) of the material. In order to be used in the parameters identification, this response should be converted in a local one (i.e. stress-strain curve). This can be simply done for homogeneous mechanical test. However, this condition may not be satisfied even for some classical mechanical tests (e.g. the simple shear test). Keeping in mind the boundary effects and the accuracy of the experimental measurement, the assumption of strain field homogeneity is commonly accepted. But, it can be also partly inspected using the full-field measurement techniques. Therefore, two identification methods can be adopted. The first one is based on a homogeneous interpretation of the mechanical test. This method requires an analytical computation of the stress state for a given strain state and viceversa. For some specific stress and strain states, this method was implemented in the SiDoLo software [Haddadi et al., 2006]. The second method doesn't require the homogeneity of the strain field. It uses an inverse identification procedure through FE computation. An example was proposed by [Flores et al., 2007] in Lagamine FE code.

In all cases, the parameters identification procedure is based on a minimization of a cost function using least squares estimation. Such cost function measures the agreement between experimental and simulated data. Different material parameters strategies can be considered, as simultaneous (yield locus and hardening laws) or sequential material parameters identification (yield locus then hardening laws). An example is proposed in Figure 2.

The stress-strain curves for uniaxial tensile test and simple shear test along the rolling direction obtained from different identification strategies are presented. The material work-hardening is described using Teodosiu-Hu model [Haddadi et al., 2006] coupled to von Mises or [Hill, 1948] criterion. Figure 2(a) presents results using inverse FE-identification. The labels indicated on the figure mean: 
(1) [Hill, 1948] using $\mathrm{r}(\alpha)$ : sequential identification strategy is adopted. First the material parameters $\{\mathrm{F}, \mathrm{G}, \mathrm{H}$ and $\mathrm{N}\}$ for [Hill, 1948] are determined using the Hill coefficients of anisotropy $\mathbf{r}_{0}, r_{45}$ and $r_{90}$ and $\sigma_{0}{ }^{2}$. Then, the material parameters of the hardening laws are determined with fixed $\{F, G, H$ and N\}.

(2) [Hill, 1948] using $\sigma(\alpha)$ and $N$ not fitted: sequential identification strategy is adopted. First the material parameters $\{F, G, H$ and $N\}$ for [Hill, 1948] are determined using the initial yield stresses for different mechanical tests Then, the material parameters of the hardening laws are determined with fixed $\{F, G, H$ and $N\}$.

(3) [Hill, 1948] using $\sigma(\alpha)$ and $N$ fitted: simultaneous identification strategy is adopted. First the material parameters $\{F, G, H$ and $N\}$ for [Hill, 1948] are determined using the initial yield stresses for different mechanical tests Then, the material parameters of the hardening laws are determined with fixed $\{\mathrm{F}, \mathrm{G}, \mathrm{H}\}$ only.

According to [Flores et al., 2007], the best fitting is obtained when simultaneous identification is adopted (Figure 2(a)). This means that the change of the yield surface during the hardening is taken into account. The identification strategy in this context proposes average values of the material parameters that describe such change.
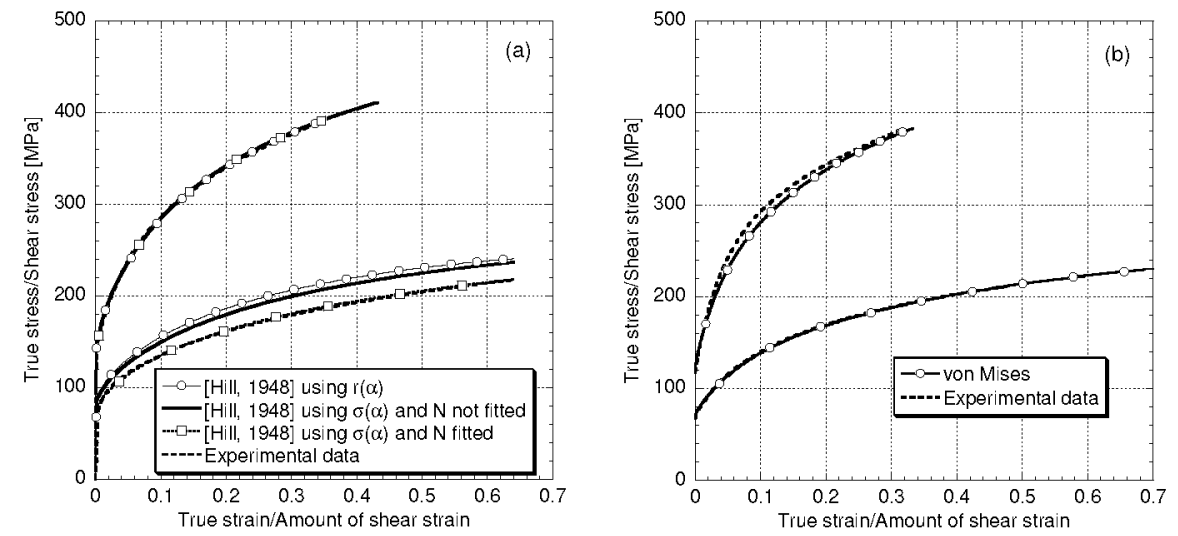

FIGURE 2. Teodosiu-Hu hardening laws identification using von Mises or [Hill, 1948] yield loci for an IF mild steel. (a) Different identification strategies proposed by [Flores et al., 2006], and (b) by

[Bouvier et al., 2003].

However for this material, a micromechanical computation shows that the contribution of the texture (i.e. the geometrical hardening) to the macroscopic hardening is rather small compared to the evolution of the microstructure (i.e. density of dislocations and their patterning...), even when significant texture evolution takes place (e.g. the simple shear test). The identification result of Figure 2(b) is obtained using direct optimization with SiDoLo software, of the hardening parameters using an isotropic von Mises yield surface for the same material of Figure 2(a).

These two strategies of identification lead to the same behavior description for the uniaxial tensile test and the simple shear test. However, the in-plane description of the

\footnotetext{
${ }^{2}$ The initial yield stress of uniaxial tensile test along the rolling direction
} 
strain anisotropy is very different (Figure 3). This leads to a significant effect on FE simulations as discussed in the previous section.

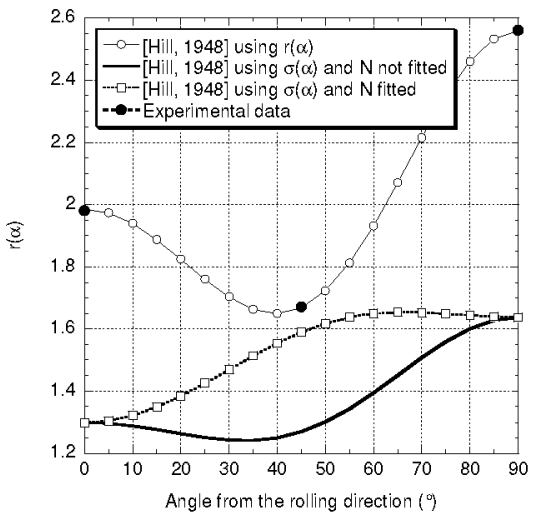

FIGURE 3. In-plane description of the strain anisotropy obtained with the identified parameters of

[Hill, 1948] yield locus after [Flores et al., 2006].

\section{SELECTION OF THE EXPERIMENTAL DATA FOR THE PARAMETERS IDENTIFICATION}

It is an important issue that the stress and/or the strain states involved in the identification strategy include the domain of intended applications.

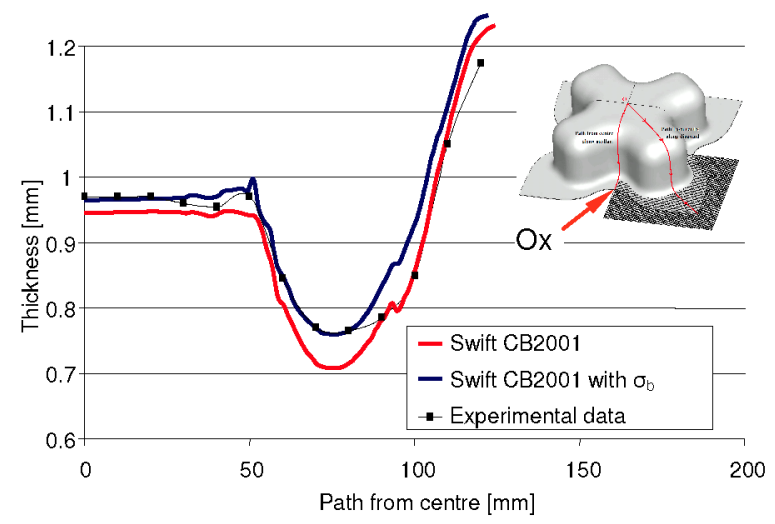

FIGURE 4. Thickness distribution along the rolling direction Ox for an aluminum alloy AA5182-O deformed up to $60 \mathrm{~mm}$ depth.

However, taking into account such considerations is not always feasible due to the difficulty in carrying out the mechanical test that reproduces the desired stress or strain fields. The cross tool test (Figure 4) was specially designed in order to impose different strain paths to the material. It is a suitable tool for the investigation of the capabilities of constitutive models. However, the question of the identification strategy should be carefully taken under consideration here. In the example of Figure 4 , the 
behavior is described by the isotropic Swift law coupled to the non quadratic criterion recently proposed by [Cazacu and Barlat, 2001]. Two identification strategies are performed using or not the data from the equibiaxial test. A significant sensitivity of the FE results in term of thickness prediction is observed using the two identification strategies. This can be explained by the presence of the biaxial stress state in this forming process.

\section{CONCLUSION}

In this paper, we investigate the problem of material parameter identification of inelastic constitutive laws in connection with FE predictions. It is worth noting that the identification procedure of constitutive laws is far from being trivial and may have a large effect on FE predictions. The following conclusion can be drawn:

(i) The question of non uniqueness solution of the identification problem may be partly solved knowing the physical significance of the material parameters involved in the constitutive laws. As an example, the sequential identification strategy discussed in the paper is based on the assumption of weak contribution of the texture evolution on the hardening. In such situation, the resort to micromechanical models may bring some clarification.

(ii) Another encounter problem in material parameter characterization concerns the absence of the experimental data. This situation occurs when the conventional mechanical tests are not possible to be performed (e.g. characterization of through thickness anisotropy for sheet material). The example discussed in section 2 (i.e. deep drawing of a cylindrical cup) clearly show that the generally adopted through-thickness isotropic behavior assumption deteriorates the FE prediction.

(iii) The identification strategy can not be completely disconnected from the simulated forming process. Indeed, a very accurate material behavior can be obtained for the experimental database involved in the identification process. However, such specific stress and/or strain states can be far from the ones involved in the simulated forming process, leading to bad prediction. An example is given with the biaxial stress state in the cross tool simulation.

Other applications are also under investigation (full-field measurement input in the identification strategy, kinemetic hardening characterization and springback sensitivity...).

\section{REFERENCES}

1. S. Bouvier, C. Teodosiu, H. Haddadi, V. Tabacaru (2003), J. Physique IV 105, pp.215-222 (2003).

2. S. Bouvier, J.L. Alves, M. Oliveira, L.F. Menezes and C. Teodosiu, in 12th International Symposium Plasticity-2006, edited by A. Khan, 2006, Halifax (Canada).

3. O. Cazacu and F. Barlat, Math. Mech. Solids 6, pp. 613-630 (2001).

4. P. Flores, L. Duchêne, C. Bouffioux, T. Lelotte, C. Henrard, N. Pernin, A. Van Bael, S. He, J. Duflou and A.M. Habraken, Int. J. Plasticity 23, 420-449 (2007).

5. H. Haddadi, S. Bouvier, M. Banu, C. Maie rand C. Teodosiu, Int. J. Plasticity 22, 2226-2271 (2006). 